\title{
Cryo-TEM Study of Interfaces in Rechargeable Lithium Batteries
}

\author{
Vahid Jabbari, Ramasubramonian Deivanayagam and Reza Shahbazian-Yassar
}

University of Illinois at Chicago, Chicago, Illinois, United States

Despite the worldwide dominance of lithium-ion batteries in consumer applications, there continue to be challenges with simultaneously improving their safety and long-term cyclability. Solid-electrolyte interphases (SEI), formed at the interface of the electrode and electrolyte, is typically composed of organic (e.g., polymers) and inorganic (e.g., oxides, carbonates, fluorides) components and play an important role in determining the long-term cycling of lithium-ion and lithium-metal batteries. Although its existence had been confirmed more than two decades ago, there has been only little understanding about its formation, favorable conditions required for its formation, and the factors determining the composition. Knowing these factors would pave ways for proposing systematic approaches to fine-tune the SEI towards enhancing the long-term cycling performance of batteries [1,2].

Formation and characterization of SEI layer is widely investigated by many chemical and surface-sensitive techniques (e.g. NMR, Raman, IR, and XPS). However, incompatibility with both solid and liquid phases, lack of morphological or structural information about SEI layer, beam-sensitivity of battery components after operation, difficulty in handling delicate SEI layer, volatility of components, and high chemical reactivity of anodes like lithium cause in big issues in utilizing these techniques to understand the SEI layer. For instance, while a mosaic SEI structure of inorganic and organic compounds has been proposed for carbonate-based electrolytes using conventional techniques, microscopic analysis showed that a layered structure is possible as well, especially in ether-based electrolytes. This shows that the existing knowledge surrounding the SEI is subject to debate and involves a lot of speculation mainly due to the limitations in characterization. Direct observation of the SEI-from its formation up to the point where it reaches maximum thickness - would also provide useful information about the nature of the structure and its composition. Yet, standard TEM and indirect-imaging are unable to preserve native nature of SEI layer (composed of chemically reactive as well as beam-sensitive components), but, under cryogenic conditions (materials are frozen by verification in liquid nitrogen), materials can retain their pristine nature. During last few years, this emerging powerful technique, called transmission electron cryomicroscopy (CryoTEM), is used to explore properties of SEI layer [1-3].

Even though new valuable insights are offered by the recent CryoTEM studies, however, these reports deal with formation and properties of SEI layer using only Li anode and a few commercially available liquid electrolyte materials (e.g. EC/DMC) and characteristic of the SEI layer in different systems is largely unknown. We aim to identify morphological, structural, and chemical properties of SEI layer formed using different anode materials (e.g. graphene) as well as electrolyte materials (e.g. composite, gel and solid polymer electrolytes) under varied conditions (e.g. low and high cycling) by CryoTEM in conjunction with EIS and other techniques (e.g. NMR, Raman, IR, and XPS). One of the main goals is to use electron energy loss spectroscopy (EELS) to analyze elemental and chemical composition of the SEI layer which is largely unknown in the previous CryoTEM reports. We also aim to synthesize some of the organic and polymeric SEI components which are not commercially available under ex-situ conditions to further expand the current knowledge on SEI layer. For instance, a common chemical compound in SEI, lithium methyl carbonate or LMC - formed as a result of degradation of DMC in the presence of Li ion under applied potential - is synthesized and the synthetic protocol and ${ }^{1} \mathrm{H}$ NMR analysis are shown in Fig. 1. Our findings can further assist in expanding the current knowledge and development of long-term cycling Li-ion batteries by engineering suitable natural and artificial SEI layers. 

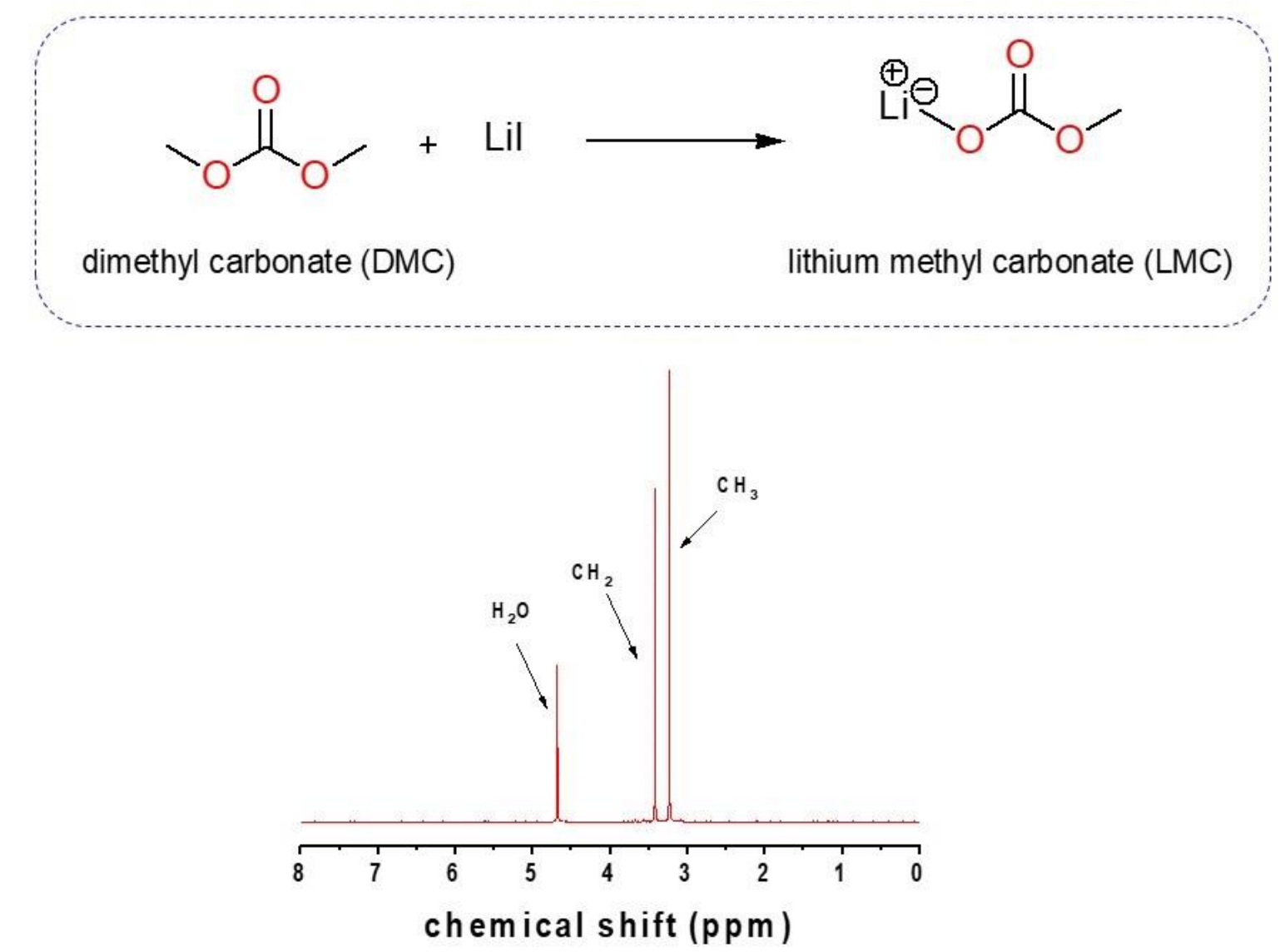

Figure 1. Synthetic protocol for LMC, and 1H NMR analysis of the as-synthesized LMC.

\section{References}

[1] J. Wang et al., Improving cyclability of Li metal batteries at elevated temperatures and its origin revealed by cryo-electron microscopy. Nature Energy 2019, 4, 664.

[2] Michael J. Zachman et al., Cryo-STEM mapping of solid-liquid interfaces and dendrites in lithiummetal batteries, Nature 2018, 560, 345.

[3] Yuzhang Li et al., Atomic structure of sensitive battery materials and interfaces revealed by cryoelectron microscopy, Science 2017, 358, 506. 